\title{
A prospective study of the early postsurgical psychiatric associations of epilepsy surgery
}

\author{
H A Ring, J Moriarty, M R Trimble
}

\begin{abstract}
Objectives- To examine prospectively the frequency and nature of psychiatric symptoms seen in patients during the first three months after temporal lobe surgery for chronic intractable epilepsy and in addition to study the relation between presurgical mental state, laterality of surgery, and postsurgical seizure and psychiatric course.

Method-A consecutive series of 60 patients being assessed for temporal lobe surgery for intractable epilepsy were studied. They were interviewed before surgery and at six weeks and again at three months after operation.

Results-At six weeks after surgery half of those with no psychopathology preoperatively had developed symptoms of anxiety or depression and $45 \%$ of all patients were noted to have increased emotional lability. By three months after surgery emotional lability and anxiety symptoms had diminished whereas depressive states tended to persist. Patients with a left hemispheric focus were more likely to experience persisting anxiety.

Conclusion-The early months after surgery for epilepsy are characterised by the relatively common presence of psychiatric symptoms. It is proposed that presurgical and early postsurgical neuropsychiatric involvement in programmes of surgery for epilepsy will help to improve the quality of the treatment package offered to patients.
\end{abstract}

(F Neurol Neurosurg Psychiatry 1998;64:601-604)

Keywords: epilepsy surgery; neuropsychiatry

Currently $5 \%-10 \%$ of patients with chronic medically refractory epilepsy may be candidates for surgical treatment. In the United Kingdom it has been estimated that there is an annual need for 600-2000 such operations. ${ }^{1}$ Although at the moment the number of operations performed each year is inadequate to meet demand, rates of surgical treatment are increasing.

The overall outcome for any patient after surgery is determined not only by the resulting seizure frequency, but also by cognitive and psychiatric state. Careful presurgical neuropsychological assessment is now an integral part of evaluation in most centres performing surgery for epilepsy. However, formal neuropsychiatric presurgical appraisal and postsurgical follow up remain rare. This is despite evidence of an increased incidence of new psychiatric symptomatology after surgery.
Accounts of psychiatric sequelae of surgery for epilepsy can be found in the Maudsley series, described by Taylor ${ }^{2}$ and more recently by Bruton. ${ }^{3}$ The Maudsley series records some patients who developed new psychosis postoperatively, as well as disclosing an increased frequency of depression. Suicide was reported in $2.4 \%$ of the sample, accounting for $22 \%$ of the postoperative deaths. In addition, findings suggest that certain presurgical psychopathology, such as a chronic florid psychosis, may not be helped by surgery ${ }^{4}$ whereas other abnormal behaviours may be ameliorated, particularly aggression $^{5}$ There is also evidence that of those undergoing surgical treatment of their epilepsy, patients with right sided temporal lobectomies may be particularly prone to more severe psychiatric disturbances. ${ }^{6}$

The aim of the present study was to prospectively determine the early psychiatric associations of surgery for epilepsy. We sought to focus on the early postoperative period for two reasons; firstly, because clinical findings suggest that patients develop psychiatric symptoms at this stage, and secondly because most of the published data report on periods starting at least six months, and often several years, after surgery. This paper presents data from the first three months of postsurgical follow up.

\section{Methods}

A consecutive series of patients with chronic treatment resistant epilepsy admitted, mostly for videotelemetry, as part of a standard presurgical assessment received a standardised psychiatric evaluation. Those proceeding to surgery were reassessed at six weeks and three months after surgery in a neuropsychiatric outpatient clinic. The psychiatric assessment was based on a clinical interview (including questions regarding peri-ictal depression and periictal psychotic phenomena). In addition, the social stress and support interview ${ }^{7}$ was employed to identify possible sources of personal or psychosocial difficulties and to categorise patients' perceptions of social, family, and financial domains of life as positive, negative, or neutral. (The study protocol also included the present state examination (PSE) ${ }^{8}$ to be used to characterise the nature of any psychotic symptoms detected during the clinical interview. None of the patients reported in this study had any psychotic symptoms so the PSE was not used.)

Based on the results of the assessment the patients were allocated to one of four diagnostic groupings according to the clinical phenomena found. Psychiatric disorders were defined according to DSM IV ${ }^{9}$ criteria, but as if there were no associated general medical condition. 
Table 1 Patient characteristics

\begin{tabular}{ll}
\hline Sex & Female $=39$ \\
Male at surgery & Range $=16-48 \mathrm{y}$ \\
& Mean (SD) =27(7.4) y \\
Laterality of surgery & Left $=40 \%$ \\
& Right $=60 \%$ \\
Surgical procedure & Temporal lobectomy = 59 patients \\
& Selective amygdalohippocampectomy \\
& $=$ one patient
\end{tabular}

Table 2 Psychiatric state at each assessment

\begin{tabular}{llll}
\hline & $\begin{array}{l}\text { Before } \\
\text { operation }\end{array}$ & $\begin{array}{l}6 \text { weeks } \\
\text { after } \\
\text { operation }\end{array}$ & $\begin{array}{l}3 \text { months } \\
\text { after } \\
\text { operation }\end{array}$ \\
\hline Seen at each assessment (n) & 60 & 60 & 52 \\
Those at each assessment in each psychological group (\%): & 48 & 26 & 48 \\
Normal & 21 & 24 & 38 \\
$\begin{array}{l}\text { Depression } \\
\text { Anxiety }\end{array}$ & 18 & 42 & 10 \\
$\begin{array}{l}\text { Psychosocial or personal } \\
\text { difficulties }\end{array}$ & 13 & 8 & 4 \\
\hline
\end{tabular}

This convention is adopted to help communicate the phenomenological nature of the psychiatric states and is not intended to convey aetiology. Patients were classified according to their predominant clinical state. If depression was present patients were considered under this diagnosis. If anxiety symptoms were pre-eminent, in the absence of significant depressive symptoms, then patients were categorised under the heading of anxiety. If, in the absence of a diagnosis of depression or anxiety, patients reported conflicts or concerns with respect to relationships with other people (for example, close friends, family, or other carers) or organisations (for example, council housing or social service departments) of sufficient severity to interfere with daily functioning or to require the intervention of a third party then these patients were considered to have psychosocial or personal difficulties.

\section{Results}

Altogether 60 patients who proceeded to temporal lobe surgery were assessed preoperatively. Table 1 shows the characteristics of the patients. At the six week postsurgical follow up 60 subjects were assessed and at three months 52 patients were seen.

At the preoperative (baseline) assessment $48 \%$ of the patients had no significant psychiatric symptoms. A major depressive disorder was found in $21 \%$ and $18 \%$ had a generalised anxiety disorder. In addition, $13 \%$ of patients had personal or psychosocial difficul-

Table 3 Number of patients, considered according to laterality of lesion, in each mental state category, at baseline, and at three months after surgery

\begin{tabular}{lcc}
\hline & $\begin{array}{l}\text { Right sided } \\
\text { pathology } \\
n(\%)\end{array}$ & $\begin{array}{l}\text { Left sided } \\
\text { pathology } \\
n(\%)\end{array}$ \\
\hline At baseline: & $14(39)$ & $14(57)$ \\
$\quad$ No psychopathology & $7(19)$ & $6(24)$ \\
Depression & $9(24)$ & $1(5)$ \\
Anxiety & $6(17)$ & $3(14)$ \\
Psychosocial & $18(55)$ & $7(35)$ \\
At three months after surgery: & $12(37)$ & $8(41)$ \\
No psychopathology & $1(4)$ & $4(18)$ \\
Depression & $1(4)$ & $1(6)$ \\
Anxiety & & \\
Psychosocial & & \\
\hline
\end{tabular}

ties not amounting to a DSM axis 1 or axis 2 psychiatric diagnosis. As time elapsed after surgery the proportions of these various mental states varied and table 2 shows the results from the psychiatric interviews at the postsurgical assessments.

\section{SUBSEQUENT ANALYSES}

Longitudinal course of the individual mental states from baseline

At six weeks after surgery half of those with no psychopathology at baseline had developed symptoms of anxiety or depression. At three months after surgery anxiety states had become less common but more patients were depressed in all groups. However, overall less psychopathology was found at three months than was noted at six weeks after surgery.

At six weeks after surgery $45 \%$ of patients were found at clinical interview to have increased emotional lability. At three months after surgery this emotional lability had become less common, by then occurring in $10 \%$.

Psychopathology at (a) baseline, and (b) three months after surgery, with respect to laterality of epileptic focus identified for surgical excision (table 3).

Altogether $60 \%$ of subjects underwent right sided surgery, with $40 \%$ receiving left sided surgery.

(a) At baseline, of those proceeding to right sided surgery, 38\% had no psychopathology whereas $62 \%$ displayed psychopathology or significant psychosocial difficulties. Of those proceeding to left sided surgery, at baseline $57 \%$ had no psychopathology whereas $43 \%$ displayed psychopathology or significant psychosocial difficulties. These rates of psychopathology were not significantly different between left and the right hemispheric groups.

(b) At three months after surgery, of those with right sided surgery, $55 \%$ had no psychopathology whereas $45 \%$ displayed psychopathology or significant psychosocial difficulties. Of those with left sided surgery, 35\% had no psychopathology whereas $65 \%$ displayed psychopathology or significant psychosocial difficulties. Again, considering overall psychopathology, there was no significant difference with respect to laterality of surgery.

Although more patients with a right sided than a left sided lesion had an anxiety disorder before surgery, this was not a significant difference $\left(\chi^{2}\right.$, Fisher's exact test, two tailed $p=0.12$ ). However, undergoing surgery resulted in a differential effect on rates of anxiety with respect to laterality of surgery. As shown in table $320 \%$ fewer of those with a right hemispheric focus were considered to be anxious at three months after surgery than during their presurgical assessment, whereas of those with a left hemispheric focus $13 \%$ more were anxious at three months after surgery than had been anxious before their operation ( $\chi^{2}$, Fisher's exact test, two tailed $\mathrm{p}<0.001$ ).

\section{Discussion}

This prospective follow up study of patients undergoing surgical excision of a temporal lobe 
epileptic focus describes what happens to presurgical mental states as time passes after surgery.

Considering the "natural history" of changes in mental state after surgery, it can be seen that preoperatively just over half the patients $(52 \%)$ displayed significant psychopathology or personality or psychosocial difficulties not amounting to a DSM axis 1 or axis 2 psychiatric diagnosis. Although this figure sounds high it should be recalled that the patients being considered for surgical treatment of their epilepsy have a chronic treatment resistant condition with frequent seizures, and therefore differ from most people with epilepsy. Our finding is consistent with data from the series reported by Jensen and Larsen ${ }^{10}$ of patients with temporal lobe epilepsy undergoing surgery, in which $85 \%$ were considered to have shown some type of psychiatric disorder between the onset of their epilepsy and the time of surgery. In a more recent Danish series 32\% of 47 patients with intractable partial epilepsy proceeding to surgery had experienced psychiatric disturbances before surgery. ${ }^{11}$

It should be noted that as well as undergoing assessment at the six week and three month clinic visits, patients also received any psychiatric treatment indicated. Hence emotional support was obtained by many of the patients and antidepressant treatment by a few. It is not possible for us to distinguish the natural history of treated from untreated postsurgical psychopathology. However, our findings indicate that changes in mental state develop in the early stages after surgery and that these changes may be severe enough to warrant psychiatric intervention.

The time of greatest psychiatric disturbance for the 60 patients reported in this paper was at six weeks after surgery. At that time about a quarter of patients $(27 \%)$ who had been free from psychopathology at baseline showed an anxiety state and a similar proportion (23\%) had become depressed. These patients could not generally describe any specific concerns that had led to their deterioration in mood.

In the early stages of this study emotional lability was not routinely assessed although it is noted that it was not spontaneously mentioned by any of the patients during their presurgical assessments. However, it became apparent to the investigators in the course of the first few six week postsurgical assessments that increased emotionality was a source of concern to some of the patients who had noticed that they were laughing or, more often, crying inappropriately. After this finding all the patients were, at their postsurgical assessments, specifically asked questions to elicit any evidence of emotional lability. (Only a minority of patients were asked these questions presurgically, hence we do not have reliable baseline data on emotional lability. However, in none of the patients that were asked presurgically was emotional lability noted.) Patients were asked if they had recently become more emotional, manifest as for instance being very easily but briefly upset or cheered up by minor events (for example, a sad television programme or receiv- ing good wishes from a friend). Respondents were clear that these responses were more excessive than their normal emotional responses. It was striking that at the six week assessment almost half of the patients were noted to have increased emotional lability, By the time three months had elapsed after surgery this emotional lability had become much less common, by then occurring in only $10 \%$ of patients. Several possible mechanisms may underlie this early postsurgical emotional lability including an emotional response to the trauma of surgery and relief at surviving it together with concerns regarding the longer term outcome, a biologically determined emotional response specifically to surgical disruption of a temporal lobe (given the well established role of medial temporal structures in the control and manifestation of emotion), or a biologically determined emotional response to some of the more general physiological effects of a neurosurgical procedure. It is not possible with the data currently available to determine which, if any, of these possibilities may account for the very obvious transient development of emotional lability in this study. Nevertheless, the frequency with which this disturbance of mental state occurred suggests that it would be reasonable to warn all patients and their families before surgery that they may experience a brief increase in emotionality in the weeks after the operation.

Less overall psychopathology was noted in all the psychological groups at three months than had been found at six weeks after surgery. In particular, anxiety developed fairly soon after surgery but tended to remit by three months. Short term postoperative anxiety was also reported after temporal lobectomy by Bladin, ${ }^{12}$ occurring in $52 \%$ of his sample but responding well to treatment. However, in our series whereas anxiety tended to remit, depression persisted and indeed became more common at three months than it had been at six weeks after surgery. In most patients the depression was of only mild to moderate severity, but nevertheless represented an important clinical issue. The reasons for this increase in the frequency of depression are unclear. Anecdotally it was noted that several patients commented that those around them now seemed to have greater expectations of them with respect to relationships, work, and other aspects of daily life. The patients largely considered that these expectations were premature while they were still largely preoccupied with fears that the surgery would in the end fail to cure them and that their seizures would return.

With respect to the nature of the interaction between mental states and laterality of surgery it was found that laterality of epileptic focus and surgical resection was not associated with differing rates or types of psychopathology before surgery, although there was a trend for those with right hemispheric pathology to be more likely to be anxious. However, there was a small but significant increase in anxiety after surgery in those patients undergoing left hemispheric surgery. Although these results are 
from a few patients, it is noteworthy that a similar finding was made by Bladin. Although that author speculated that postoperative clonazepam withdrawal may have contributed to the development of anxiety in his patients, in our series this is not a possible explanation. In the literature the clearest association between laterality of focus and abnormalities in mental state after surgery is with the presence of psychosis. ${ }^{4}$ In this series no patients were psychotic, either at baseline or in the first three months after surgery.

In conclusion, temporal lobe surgery for epilepsy was, in our study population, associated with the early but generally brief development of emotional lability, anxiety, and depression, occurring in those without mental state abnormalities at baseline as well as in those with preexisting psychopathology. Of these psychiatric states the depression was the most persistent, tending to be a problem at three months after surgery.

Our sample size was small and one of the study findings, that of emotional lability, was not introduced until the first few postsurgical assessments had indicated that this may be a clinically important issue. Nevertheless, the results of this prospective follow up of early postsurgical mental states indicate that psychopathology during this period is relatively common. We suggest, on the basis of these findings, that a neuropsychiatric contribution to the presurgical assessment and postsurgical care of patients undergoing surgical treatment of epilepsy is appropriate for the optimal management of these patients. It is now our own practice to discuss possible changes in mental state with patients, warning that they may occur and providing reassurance that if they do then they are generally brief, with therapeutic interventions available if needed.

1 Duncan JS, Shorvon SD, Fish DR. Clinical epilepsy. New York: Churchill Livingstone, 1995:349.

2 Taylor DC. Mental state and temporal lobe epilepsy. A corTaylor DC. Mental state and temporal lobe epilepsy. A cor-
relative account of 100 patients treated surgically. Epilepsia 1972;13:727-65.

3 Bruton CJ. The neuropathology of temporal lobe epilepsy. Oxford: Maudsley Monographs, Oxford University Press, 1988:

4 Trimble MR. The psychoses of epilepsy. New York: Raven Press, 1991.

5 Walker EA, Blumer D. Behaviour effects of temporal lobectomy. In: Blumer D, ed. Psychiatric aspects of epilepsy. Wash.

6 Mace CJ, Trimble MR. Psychosis following temporal lobe surgery: a report of six cases. $\mathcal{F}$ Neurol Neurosurg Psychiatry surgery: a report of

7 Jenkins R, Mann AH, Belsey E. The background, design and use of a short interview to assess social stress and support in research and clinical settings. Soc Sci Med 1981;15: 195-203.

8 Wing JK, Cooper JE, Sartorius N. The measurement and classification of psychiatric symptoms. Cambridge: Cambridge University Press, 1974

9 American Psychiatric Association. Diagnostic and statistical manual of mental disorders, 4th ed. (DSM-IV) Washington, DC: APA, 1994.

10 Jensen I, Larsen JK. Mental aspects of temporal lobe epilepsy: follow up of 74 patients after resection of a epilepsy: follow up of 74 patients after resection of a
temporal lobe. $\mathcal{F}$ Neurol Neurosurg Psychiatry 1979;42:25665.

11 Naylor AS, Rogvi-Hansen B, Kessing L, et al. Psychiatric morbidity after surgery for epilepsy: short term follow up of patients undergoing amygdalohippocampectomy. 7 Neurol Neurosurg Psychiatry 1994;57:1375-81.

12 Bladin PF. Psychosocial difficulties and outcome after temporal lobectomy. Epilepsia 1992;33:898-907. 